\title{
Nonrelativistic exchange-energy density and exchange potential in the lowest order of the $1 / Z$ expansion for ten-electron atomic ions
}

\author{
I. A. Howard, ${ }^{1}$ N. H. March, ${ }^{2}$ P. Senet, ${ }^{1}$ and V. E. Van Doren ${ }^{1}$ \\ ${ }^{1}$ Department of Physics, University of Antwerp (RUCA), Groenenborgerlaan 171, B-2020 Antwerp, Belgium \\ ${ }^{2}$ Oxford University, Oxford, England
}

(Received 23 June 2000; published 15 November 2000)

\begin{abstract}
The Dirac exchange energy $E_{x}$ has a density $\varepsilon_{x}(r)$ in a spherically symmetric ten-electron atomic ion that is determined by the idempotent first-order density matrix $(1 \mathrm{DM})$. In turn, this $1 \mathrm{DM}$ has as its leading term in the $1 / Z$ expansion, with $Z$ the atomic number, the bare Coulomb result. This latter quantity is known analytically from the study of March and Santamaria [Phys. Rev. A 38, 5002 (1988)]. Here $\varepsilon_{x}(r)$ is calculated analytically, and presented graphically for $Z=92$ in this large- $Z$ limit. The Slater approximation to the exchange potential $V_{x}(\mathbf{r})$, namely, $V_{\mathrm{Sla}}(\mathbf{r})=2 \varepsilon_{x}(r) / \rho(r)$ with $\rho(r)$ the ground-state electron density, is also plotted for $Z=92$. In the large- $Z$ limit, $V_{x}(\mathbf{r})$ can be obtained by functional differentiation of the resultant exchange energy, and is expressed in terms of electron and kinetic-energy densities. Numerical calculations, based on $V_{\mathrm{Sla}}(\mathbf{r})$ plus approximate corrections, are also presented.
\end{abstract}

PACS number(s): 31.15.Ew

\section{BACKGROUND AND OUTLINE}

Though density-functional theory (DFT) is now a widely used technique in the many-electron problem encountered in atoms, molecules, and clusters, important problems remain in constructing the exchange-correlation potential $V_{\mathrm{xc}}(\mathbf{r})$ (see, for example, Ref. [1]). Therefore, Holas and March [2] gave a complete theory of $V_{\mathrm{xc}}(\mathbf{r})$ based on low-order density matrices. This has moved attention to the construction of such density matrices, though of course it is recognized that eventually, to complete the DFT approach, one will require these low-order density matrices as functionals of the "diagonal" ground-state density $\rho(\mathbf{r})$. In the present paper, attention will be focused entirely on the first-order density matrix (1 DM) $\gamma\left(\mathbf{r}, \mathbf{r}^{\prime}\right)$. This is the natural starting point for the calculation of kinetic-energy density, $t(\mathbf{r})$, for instance, since the total kinetic energy $T$ can be written as

$$
T=\left.\left(\hbar^{2} / 2 m\right) \int \boldsymbol{\nabla}_{r} \cdot \nabla_{r^{\prime}} \gamma\left(\mathbf{r}, \mathbf{r}^{\prime}\right)\right|_{r^{\prime}=r} d \mathbf{r}
$$

Though $t(\mathbf{r})$ is not defined uniquely by the result (1.1), one acceptable definition is plainly

$$
t(\mathbf{r})=\left.\left(\hbar^{2} / 2 m\right) \boldsymbol{\nabla}_{r} \cdot \nabla_{r^{\prime}} \gamma\left(\mathbf{r}, \mathbf{r}^{\prime}\right)\right|_{r^{\prime}=r}
$$

What is important for the present paper is that, in the framework provided in the early work of Dirac [3], the exchange energy $E_{x}$ is also determined by the (now idempotent) firstorder density matrix $\gamma\left(\mathbf{r}, \mathbf{r}^{\prime}\right)$. Thus, in a spin-compensated system on which all attention will be focused below, the idempotency condition reads [for the diagonal density $\rho(\mathbf{r})$ ]

$$
\rho(\mathbf{r}) / 2=\int\left\{\gamma\left(\mathbf{r}, \mathbf{r}^{\prime}\right) / 2\right\}^{2} d \mathbf{r}^{\prime}
$$

Naturally, however, this idempotency condition precludes inclusion of electron correlation, for which the equality (1.3) has to be replaced by a (much weaker) condition in the form of an inequality.

Even at the idempotent level, however, the Dirac exchange energy [3]

$$
E_{x}=-\left(e^{2} / 4\right) \int \frac{\gamma^{2}\left(\mathbf{r}, \mathbf{r}^{\prime}\right)}{\left|\mathbf{r}-\mathbf{r}^{\prime}\right|} d \mathbf{r} d \mathbf{r}^{\prime}
$$

is not easy to turn into DFT form for the reason already stated above, namely, that one needs the first-order idempotent density matrix $\gamma\left(\mathbf{r}, \mathbf{r}^{\prime}\right)$ as a functional of the diagonal ground-state density $\rho(\mathbf{r})$. Therefore, in the present paper, we shall combine the Dirac result (1.4) with the important $1 / Z$ expansion of atomic theory, which was first connected fundamentally with DFT in the work of March and White [4]. These authors were concerned with the nonrelativistic ground-state energy of a heavy atomic ion having nuclear charge Ze and $\mathrm{N}$ electrons, which takes the form (going back to Hylleraas [5] and especially developed later by Layzer and co-workers [6]):

$$
\begin{aligned}
E(Z, N)= & Z^{2}\left[\varepsilon_{0}(N)+(1 / Z) \varepsilon_{1}(N)\right. \\
& \left.+\cdots+\left(1 / Z^{n}\right) \varepsilon_{n}(N)+\cdots\right] .
\end{aligned}
$$

The main result of March and White [4] was to demonstrate, by bringing Eq. (1.5) into contact with the original form of DFT valid for large $Z$ and $N$ (namely, the Thomas-Fermi statistical theory [7]), that the asymptotic form of the coefficients $\varepsilon_{n}(N)$ for large $N$ was given by

$$
\varepsilon_{n}(N) \approx N^{n+1 / 3}
$$

a result subsequently confirmed in a more rigorous study of Tal and Levy [8].

However, the point to be exploited in the present paper is that the leading term in the $1 / Z$ expansion of the total energy $E(Z, N)$ of an atomic ion displayed in Eq. (1.5) is completely 
determined by the properties of the bare Coulomb potential $\left(-Z e^{2} / r\right)$. And for a closed-shell atom with filled $k$ and $l$ shells, March and Santamaria [9] have obtained the firstorder idempotent density matrix in this bare Coulomb limit in closed form as

$$
\gamma\left(\mathbf{r}, \mathbf{r}^{\prime}\right)=\rho\left(\frac{r+r^{\prime}}{2}\right)+\left|\mathbf{r}-\mathbf{r}^{\prime}\right|^{2} F\left(\frac{r+r^{\prime}}{2}\right)
$$

Here the authors show that $F$ is related to the kinetic-energy density $t(r)$ by

$$
F=\rho^{\prime \prime} / 24-m t(r) / 4 \hbar^{2} .
$$

We record the explicit forms of $\rho(r)$ and $F(r)$ in Appendix A. Clearly, therefore, for this ten-electron problem, i.e., a nonrelativistic Ne-like atomic ion in the limit of large $Z$, one can evaluate the Dirac exchange energy $E_{x}$ from Eq. (1.4) in this large- $Z$ limit by inserting the Coulomb density matrix (1.7).

The outline of the paper is then as follows. In Sec. II immediately below, it will be shown that the exchange energy density $\varepsilon_{x}(r)$ can be evaluated in closed form using Eqs. (1.4) and (1.7) in this large- $Z$ limit for ten-electron atomic ions, the natural definition of $\varepsilon_{x}(r)$ following from Eq. (1.4) as

$$
\varepsilon_{x}(\mathbf{r})=-\left(e^{2} / 4\right) \int \frac{\gamma^{2}\left(\mathbf{r}, \mathbf{r}^{\prime}\right)}{\left|\mathbf{r}-\mathbf{r}^{\prime}\right|} d \mathbf{r}^{\prime}
$$

The resulting (spherically symmetric) exchange energy density will also be presented graphically there for the case $Z$ $\approx 92$, though, of course, it is recognized that for any subsequent contact of all the results with experiment, relativistic corrections will be required for such a uranium highly charged positive ion. Section III then returns to one focal point of current DFT, namely, the potential $V_{x}(\mathbf{r})$ representing exchange. Of course, in our use of Dirac's result (1.4), we are restricted to idempotent $1 \mathrm{DM}$ 's, and therefore correlation is neglected. It is known, however, that exchange enters before correlation, from the work of Scott for exchange [10] and various later workers for correlation, in the spirit of the $1 / Z$ expansion of the total energy in Eq. (1.5), but now with partially summed subseries as in the Thomas-FermiDirac (TFD) statistical theory (March and White [4]; see also March and Parr [11]). For example, for neutral atoms, Scott showed that $E_{x}^{\mathrm{TFD}} \propto Z^{5 / 3}$, whereas there is agreement that $E_{\text {correlation }}$ depends on a lower power of $Z$; proposals have been made for $Z^{n}$ with $n=1$ or $4 / 3$ (March and Wind [12], Kais, Sung, and Herschbach [13]). In Sec. III, an exact functional differentiation of the exchange energy in the large- $Z$ limit is reported, the answer for $V_{x}(\mathbf{r})$ involving both electron and kinetic-energy densities. Then in Sec. IV, the approximation for $V_{x}(\mathbf{r})$ proposed by Slater is examined analytically and is also displayed numerically for $Z=92$. It will be argued that the Slater potential is already a useful approximation to the full exchange potential, though it is not quantitative near the atomic nucleus. Corrections are exhibited from the early work of Harbola and Sahni [14], modifications to which have been treated by Holas and March [2] (see also Levy and March [15]). Section V constitutes a summary with some proposals for future work. Finally, in Appendix B, Scott's calculation of the neutral atom exchange energy in the TFD theory is complemented by treating the highly ionized limit corresponding in the present study to $N=10$ and large $Z$.

\section{FORM OF THE EXCHANGE ENERGY DENSITY $\varepsilon_{x}(r)$ FOR TEN-ELECTRON ATOMIC IONS IN THE (BARE COULOMB) LIMIT OF LARGE ATOMIC NUMBER $Z$}

The reduced first-order density matrix of March and Santamaria [9] [Eq. (1.7)] can be used directly in Eq. (1.9) to evaluate the exchange energy density. Since the functions $\rho\left(\left(r+r^{\prime}\right) / 2\right)$ and $F\left(\left(r+r^{\prime}\right) / 2\right)$ in Eq. (1.7) are spherically symmetric, the angular integrations in Eq. (1.9) can easily be done, taking $\mathbf{r}$ to lie along the $z$ axis, so that

$$
\begin{aligned}
\varepsilon_{x}(r)= & -\left(\pi e^{2} / 2 r\right)\left\{\int \rho^{2}\left(\left(r+r^{\prime}\right) / 2\right)\left[\left(r+r^{\prime}\right)-\left|r-r^{\prime}\right|\right] d r^{\prime}\right. \\
& +(2 / 3) \int r^{\prime} \rho\left(\left(r+r^{\prime}\right) / 2\right) F\left(\left(r+r^{\prime}\right) / 2\right)\left[\left(r+r^{\prime}\right)^{3}\right. \\
& \left.-\left|r-r^{\prime}\right|^{3}\right] d r^{\prime}+(1 / 5) \int r^{\prime} F^{2}\left(\left(r+r^{\prime}\right) / 2\right)\left[\left(r+r^{\prime}\right)^{5}\right. \\
& \left.\left.-\left|r-r^{\prime}\right|^{5}\right] d r^{\prime}\right\} .
\end{aligned}
$$

We shall return to the functional properties of Eq. (2.1) in Sec. III below. For the present, using the explicit forms of $F$ and $\rho$ given in Eqs. (A1) and (A2), respectively, we note that after radial integration, the resulting total exchange energy density $\varepsilon_{x}(r)$ is found to be

$$
\begin{aligned}
\varepsilon_{x}(r)= & (1 / 15552)\left(e^{2} \alpha^{3} / \pi r\right) \exp (-4 \alpha r)\left\{15552(1+\alpha r)+\exp (\alpha r)\left[4096+3840(\alpha r)+2304(\alpha r)^{2}+3456(\alpha r)^{3}\right]\right. \\
& +\exp (2 \alpha r)\left[-13608+2430(\alpha r)+2916(\alpha r)^{2}+1944(\alpha r)^{3}+486(\alpha r)^{4}+243(\alpha r)^{5}\right]-4096 \exp (5 \alpha r / 2) \\
& \left.+\exp (3 \alpha r)\left[-1944-972 \alpha r-972(\alpha r)^{2}\right]\right\},
\end{aligned}
$$




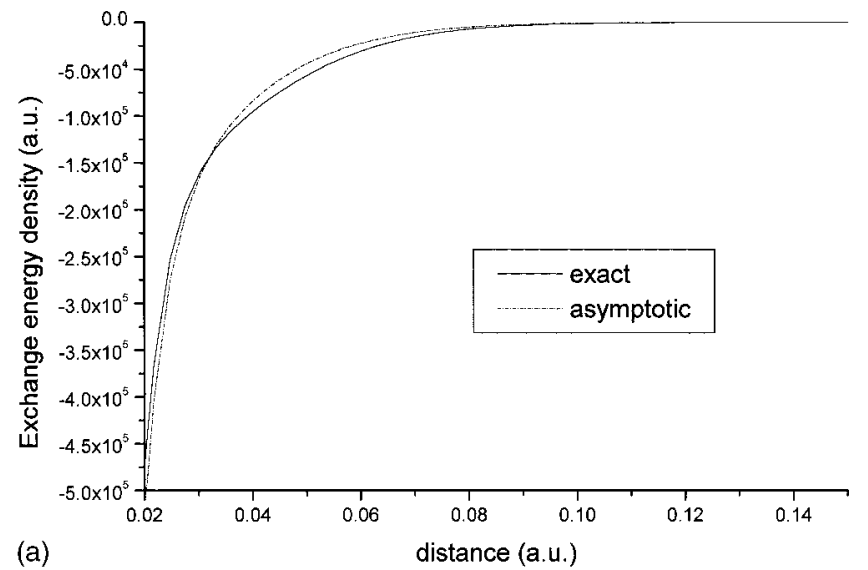

(a)

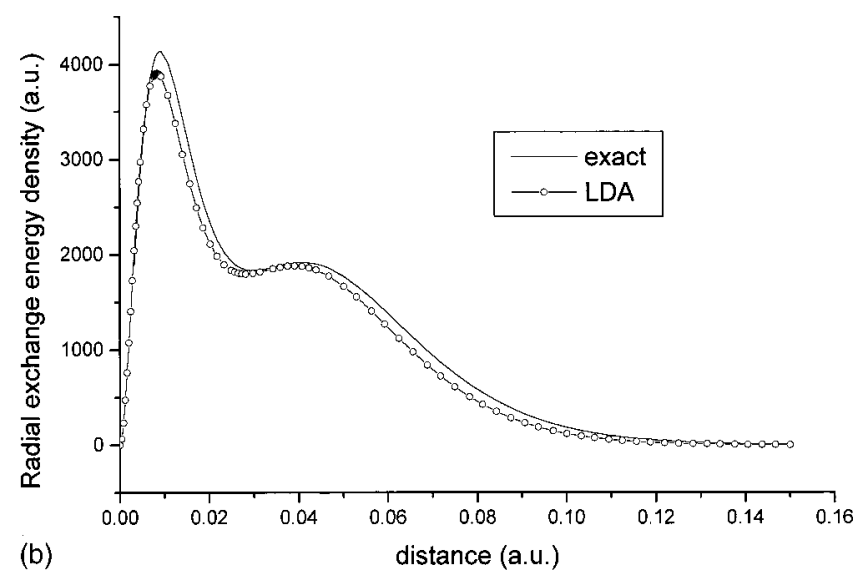

FIG. 1. (a) Exchange energy density $\varepsilon_{x}(r)$ defined by Eqs. (1.7) and (1.9), for $Z=92$. For comparison, the asymptotic form $\varepsilon_{x}^{\mathrm{AS}}(r)$ $=-e^{2} \rho(r) / r$ of the exchange energy density is also plotted. (b) Radial exchange energy density $4 \pi r^{2} \varepsilon_{x}(r)$, compared to that for the LDA, with $\varepsilon_{x}^{\mathrm{LDA}}(r)=-c_{x}\{\rho(r)\}^{4 / 3}, c_{x}=(3 / 4)(3 / \pi)^{1 / 3} e^{2}$, and $\rho(r)$ given by Eq. (A2). The total exchange energies in the limit of $Z=92$ are $E_{x}=-160.805$ and $E_{x}^{\mathrm{LDA}}=-147.969$ a.u.

where $\alpha=\left(Z / a_{0}\right)$. In spite of the $e^{2} / r$ factor outside the curly brackets, it is readily verified that $\varepsilon_{x}(0)$ is finite. This expression (2.2) is then readily integrated over $\mathbf{r}$ to yield the total exchange energy,

$$
E_{x}=-1.74788 Z e^{2} / a_{0}
$$

(see also Appendix C).

For our case of the $Z=92$, ten-electron ion, the exchange energy density $\varepsilon_{x}(r)$ is plotted in Fig. 1(a); for comparison we show, in Fig. 1(a), the asymptotic form, $\varepsilon_{x}^{\mathrm{AS}}(r)=$ - $(1 / 2) e^{2} \rho(r) / r$, to which $\varepsilon_{x}(r)$ tends at large $r$. It can be seen from Fig. 1 that the asymptotic form takes over when $r \simeq 0.08$ a.u. In Fig. $1 b$ the radial exchange energy density $4 \pi r^{2} \varepsilon_{x}(r)$ is plotted for $Z=92$ again and compared with the local-density approximation (LDA). The point-point agreement between exact and LDA in Fig. 1b is excellent. But even so, the total exchange energy $E_{x}$ from exact and LDA calculations is -160.805 and -147.969 a.u., respectively.

\section{FUNCTIONAL DIFFERENTIATION OF EXCHANGE ENERGY: THE EXCHANGE POTENTIAL FOR TEN-ELECTRON ATOMIC IONS IN THE LARGE- $Z$ LIMIT}

The purpose of this section is to show that an exact expression for the functional derivative $\delta E_{x} / \delta \rho(\mathbf{r})$, namely, the exchange potential $V_{x}(\mathbf{r})$, can be obtained from the calculation of the exact energy $E_{x}$. To do this it is useful to divide $E_{x}$ into the sum of three contributions. Returning to Eq. (2.1), we first note that the total exchange energy $E_{x}$ follows as the sum of three terms:

$$
E_{x}=E_{x}^{(1)}+E_{x}^{(2)}+E_{x}^{(3)}
$$

These terms are evaluated in Appendix C.

From the above, it follows that the total exchange energy $E_{x}$ takes the form

$$
\begin{aligned}
E_{x}= & -e^{2}\left[A_{1} \int_{0}^{\infty} d s \rho^{2}(s) s^{4}+A_{2} \int_{0}^{\infty} d s \rho(s) F(s) s^{6}\right. \\
& \left.+A_{3} \int_{0}^{\infty} d s F^{2}(s) s^{8}\right]
\end{aligned}
$$

with $A_{1}=20 \pi^{2} / 3, A_{2}=224 \pi^{2} / 9$, and $A_{3}=32 \pi^{2}$. But from Ref. [9], $F(r)$ can be related to $\rho(r)$ and the kinetic-energy density $t(r)$ by Eq. (1.8) already quoted above. Substitution of this result for $F(s)$ in Eq. (3.2) gives the total exchange energy $E_{x}$ solely in terms of $\rho(r)$ and its second derivative, plus the kinetic-energy density $t(r)$ (for details see Appendix C). Naturally, this is a result exact only in the bare Coulomb limit of the $1 / Z$ expansion considered throughout this paper. In another model, of a metal surface, a related finding has recently been reported [16]. It was stressed by March and Santamaria [17] that it was to be expected that (nonlocal generalizations of) exchange and kinetic-energy densities should be intimately related (see also Lee, Lee, and Parr [18], and a very recent study by Chan and Handy [19]).

To proceed further, let us write in a little more detail the results obtained for the functional derivatives of $E_{x}^{(i)}, i=1$ -3 . Thus,

$$
\begin{aligned}
\delta E_{x}^{(1)} / \delta \rho(\mathbf{r}) & \\
= & -\left(5 \pi e^{2} / 3\right) \int_{0}^{2 \pi} d \phi \int_{0}^{\pi} d \theta \sin \theta \int_{0}^{\infty} d s s^{4} \partial / \partial \alpha[\rho(s) \\
& \left.+\alpha \delta\left(\phi-\phi^{\prime}\right) \delta\left(\cos \theta-\cos \theta^{\prime}\right) \delta(s-r) / s^{2}\right]^{2}
\end{aligned}
$$

After some manipulation, one reaches the desired result

$$
\delta E_{x}^{(1)} / \delta \rho(\mathbf{r})=-\left(10 \pi e^{2} / 3\right) r^{2} \rho(r) .
$$

Proceeding to treat $E_{x}^{(2)}$ similarly, one finds 


$$
\begin{aligned}
\delta E_{x}^{(2)} / \delta \rho(\mathbf{r})= & \left(56 \pi e^{2} / 9\right) r^{4} F(r) \\
& +\left(224 \pi^{2} e^{2} / 9\right) \int_{0}^{\infty} d s \rho(s) s^{6} \delta F(s) / \delta \rho(\mathbf{r}) .
\end{aligned}
$$

After further manipulation and introduction of the relation between $F, \rho^{\prime \prime}$, and kinetic-energy density $t(\mathbf{r})$ in Eq. (1.8), one reaches the result

$$
\begin{aligned}
\delta E_{x}^{(2)} / \delta \rho(\mathbf{r})= & \left(56 \pi e^{2} / 9\right) r^{4} F(r)-\left(224 \pi^{2} e^{2} m / 27 \hbar^{2}\right) \\
& \times \int_{0}^{\infty} d s \rho(s) s^{6}[\delta t(s) / \delta \rho(\mathbf{r})] \\
& +\left[\left(70 \pi e^{2} / 9\right) r^{2} \rho(r)+\left(28 \pi e^{2} / 9\right) r^{3} \rho^{\prime}(r)\right. \\
& \left.+\left(7 \pi e^{2} / 27\right) r^{4} \rho^{\prime \prime}(r)\right] .
\end{aligned}
$$

Thus this part of the exchange potential can also be obtained formally exactly, but now the functional derivative of the kinetic-energy density appears. To date, we have not succeeded in calculating this term explicitly, since $T[\rho]$ is not yet known in this large- $Z$ limit. One part of $T[\rho]$, that from the $s(l=0)$ states, has been given earlier by one of us $[20]$ as

$$
T_{s}[\rho]=(1 / 4) \int\left(\rho / r^{2}\right) d \mathbf{r}
$$

and hence

$$
\delta t_{s}(r) / \delta \rho\left(\mathbf{r}^{\prime}\right)=4 \pi \delta\left(\mathbf{r}-\mathbf{r}^{\prime}\right) / r^{2} .
$$

However, no such closed result has been found for the $p$ term.

The third contribution $\delta E_{x}^{(3)} / \delta \rho(\mathbf{r})$ to the exchange potential can be written by similar arguments as

$$
\delta E_{x}^{(3)} / \delta \rho(\mathbf{r})=-64 \pi^{2} e^{2} \int_{0}^{\infty} d s s^{8} F(s) \delta F / \delta \rho(\mathbf{r}) .
$$

We are then led to the final form

$$
\begin{aligned}
\delta E_{x}^{(3)} / \delta \rho(\mathbf{r})= & \left(64 \pi^{2} e^{2} m / 3 \hbar^{2}\right) \int_{0}^{\infty} d s s^{8} F(s)[\delta t(s) / \delta \rho(\mathbf{r})] \\
& -\left(112 \pi e^{2} / 3\right) r^{4} F(r)-\left(32 \pi e^{2} / 3\right) r^{5} F^{\prime}(r) \\
& -\left(2 \pi e^{2} / 3\right) r^{6} F^{\prime \prime}(r) .
\end{aligned}
$$

Thus, the exchange potential for this ten-electron system in the large- $Z$ limit can be obtained analytically by direct functional differentiation of the exchange energy $E_{x}$, the answer being in terms of the ground-state density $\rho(r)$ and its derivatives, plus the functional derivative $\delta t(s) / \delta \rho(\mathbf{r})$ of the kinetic-energy density. This is therefore a formally complete density-functional theory of the exchange potential in this closed- $(k+l)$ shell atomic ion in the large- $Z$ limit.

Because of the absence, to date, of a calculable form of $\delta t(s) / \delta \rho(\mathbf{r})$, we follow this exact theoretical analysis by

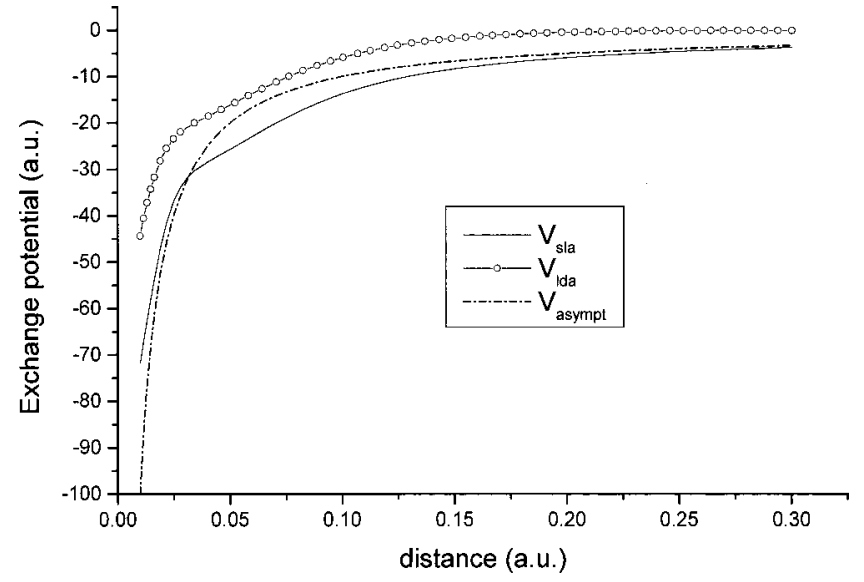

FIG. 2. Slater potential $V_{\mathrm{Sla}}(r)=2 \varepsilon_{x}(r) / \rho(r)$ constructed from $\varepsilon_{x}(r)$ as in Fig. 1 and $\rho(r)$ in Eq. (A2). $V_{\mathrm{Sla}}(r)$ has the exact asymptotic form $-e^{2} / r$ of the exchange potential $V_{x}(r)$ at sufficiently large $r$. The LDA potential, $V_{\mathrm{LDA}}(r)=-(3 / \pi)^{1 / 3} e^{2} \rho^{1 / 3}(r)$, is also shown for comparison.

reporting some approximate numerical results for $V_{x}(\mathbf{r})$, which we conveniently build around the Slater potential $V_{\text {Sla }}(\mathbf{r})$ defined immediately below.

\section{APPROXIMATE NUMERICAL RESULTS FOR EXCHANGE POTENTIAL $V_{x}(\mathbf{r})$ AND FORCE}

Given Eq. (2.2) above, one can immediately find the Slater approximation to the exchange potential, $V_{\mathrm{Sla}}(\mathbf{r})$ $=2 \varepsilon_{x}(r) / \rho(r)$, with $\rho(r)=\left.\gamma\left(\mathbf{r}, \mathbf{r}^{\prime}\right)\right|_{r^{\prime}=r}$. Figure 2 shows $V_{\mathrm{Sla}}(\mathbf{r})$ for the present $Z=92$, ten-electron case as it approaches the asymptotic limit, $V_{x}^{\mathrm{AS}}(\mathbf{r})=-e^{2} / r$ as $r \rightarrow \infty$.

In the present case of a noninteracting electronic system, the Harbola-Sahni exchange potential [14] can be expressed as the sum of the Slater potential plus a "correction" term:

$$
V_{\mathrm{HS}}(r)=V_{\mathrm{Sla}}(r)-\int_{r^{\prime \prime}=\infty}^{r} \int \frac{\left[\partial \rho_{x} / \partial r^{\prime \prime}\right]}{\left|\mathbf{r}^{\prime \prime}-\mathbf{r}^{\prime}\right|} d^{3} r^{\prime} d r^{\prime \prime},
$$

where $\rho_{x}\left(r^{\prime}, r^{\prime \prime}\right)=\gamma^{2}\left(r^{\prime}, r^{\prime \prime}\right) / 2 \rho\left(r^{\prime \prime}\right)$ is the exchange hole density. The correction term has been evaluated numerically for our case, and the resulting exchange potential $V_{\mathrm{HS}}(r)$ plotted in Fig. 3. For comparison, the Slater potential $V_{\mathrm{Sla}}(r)$ is also shown. As can be seen, the difference between the exact and the Slater potentials decreases rapidly with $r$. We mention in this context the observation of Kleinman [21] that the Slater potential is a partial functional derivative of the exchange energy with respect to charge density with wave function phases held constant. The phases must contribute to the full functional derivative near the nucleus; this contribution being short ranged, since the Slater potential has the correct asymptotic form.

It is instructive to return to the original form [14] of the Harbola-Sahni potential,

$$
V_{\mathrm{HS}}(\mathbf{r})=-\int_{r^{\prime \prime}=\infty}^{r} \int \frac{\gamma^{2}\left(\mathbf{r}^{\prime \prime}, \mathbf{r}^{\prime}\right)\left(\mathbf{r}^{\prime \prime}-\mathbf{r}^{\prime}\right)}{2 \rho\left(\mathbf{r}^{\prime \prime}\right)\left|\mathbf{r}^{\prime \prime}-\mathbf{r}^{\prime}\right|^{3}} d^{3} r^{\prime} d \mathbf{r}^{\prime \prime}
$$


and calculate the corresponding forces for our case. In particular, one can calculate $\left[\rho(r) r \partial V_{\mathrm{HS}}(r) / \partial r\right]$ and verify that the (negative of the) integral over all space recovers the total exchange energy [the Levy-Perdew equality of Eq. (A5)]. Expressing $\gamma\left(\mathbf{r}, \mathbf{r}^{\prime}\right)$ in terms of $\rho$ and $F$ and performing the angular integrations, one finds that

$$
\begin{aligned}
\rho(r) r \frac{\partial V_{\mathrm{HS}}}{\partial r}= & (\pi / r) \int_{r^{\prime}=0}^{\infty} \rho^{2}\left(\left(r+r^{\prime}\right) / 2\right)\left[\frac{\left(r-r^{\prime}\right)}{\left|r-r^{\prime}\right|}+1\right] r^{\prime 2} d r^{\prime} \\
& +(2 \pi / 3 r) \int_{r^{\prime}=0}^{\infty} \rho\left(\left(r+r^{\prime}\right) / 2\right) F\left(\left(r+r^{\prime}\right) / 2\right)\left[-\left|r-r^{\prime}\right|\left(2 r^{2}-r^{\prime 2}-r r^{\prime}\right)+\left(r+r^{\prime}\right)\left(2 r^{2}-r^{\prime 2}+r r^{\prime}\right)\right] r^{\prime} d r^{\prime} \\
& +(\pi / 15 r) \int_{r^{\prime}=0}^{\infty} F^{2}\left(\left(r+r^{\prime}\right) / 2\right)\left[-\left|r-r^{\prime}\right|^{3}\left(4 r^{2}-r^{\prime 2}-3 r r^{\prime}\right)+\left(r+r^{\prime}\right)^{3}\left(4 r^{2}-r^{\prime 2}+3 r r^{\prime}\right)\right] r^{\prime} d r^{\prime}
\end{aligned}
$$

Volume integration then gives

$$
\begin{aligned}
-E_{x}= & \int \rho(r) r \frac{\partial V_{\mathrm{HS}}}{\partial r} d^{3} r \\
= & \int_{0}^{\infty} 4 \pi^{2} r d r \int_{r^{\prime}=0}^{\infty} \rho^{2}\left(\left(r+r^{\prime}\right) / 2\right) \\
& \times\left[\frac{\left(r-r^{\prime}\right)}{\left|r-r^{\prime}\right|}+1\right] r^{\prime 2} d r^{\prime} \\
& +[\text { the last two terms from Eq.(4.3)], }
\end{aligned}
$$

where $E_{x}=-160.805$ a.u. as before. Thus, $V_{\mathrm{HS}}(r)$ satisfies one of the conditions on the exact exchange potential, although this is not a proof that $V_{\mathrm{HS}}=V_{\text {exact }}$.

The exchange force resulting from $V_{\mathrm{HS}}(r)$ is purely radial, $F_{\mathrm{HS}}(r)=-\partial V_{\mathrm{HS}}(r) / \partial r$; using Eq. (4.3), one can find analytically the force plotted in Fig. 4, where $F_{\text {Sla }}(r)$ $=-\partial V_{\mathrm{Sla}}(r) / \partial r$ is shown for comparison. Note that both $F_{\text {Sla }}(r)$ and $F_{\mathrm{HS}}(r)$ go to zero at the nucleus $r=0$.

\section{SUMMARY AND PROPOSED FUTURE DIRECTIONS}

The main achievements of the present paper are as follows:

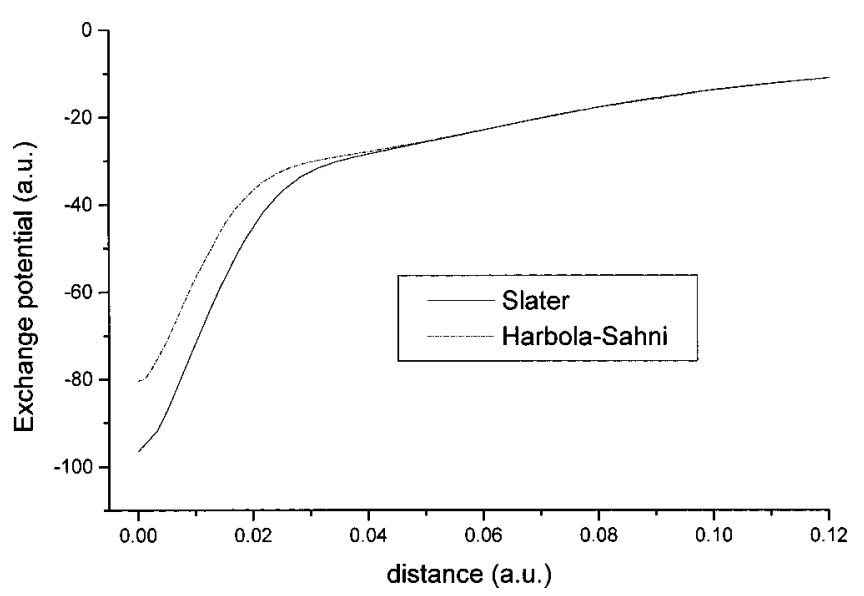

FIG. 3. The Harbola-Sahni exchange potential $V_{\mathrm{HS}}(r)$ compared with $V_{\mathrm{Sla}}(r)$. (i) In the spirit of the $1 / Z$ expansion written explicitly for atomic ions in Eq. (1.5), the limiting form of the exchange energy density $\varepsilon_{x}(r)$ at large $Z$ has been obtained (Eq. 2.2) in closed analytic form for nonrelativistic ten-electron ions.

(ii) By integration, the total exchange energy $E_{x}$ has been shown for these ten-electron ions to be proportional to $Z$ as $Z \rightarrow \infty$, with the proportionality coefficient $-1.74788\left(e^{2} / a_{0}\right)$ [see Eq. (2.3) and Appendix C].

(iii) The Slater approximation to the exchange potential $V_{x}(r)$, namely, $2 \varepsilon_{x}(r) / \rho(r)$, has hence been found analytically and is plotted for $Z=92$ in Fig. 2 .

(iv) The Harbola-Sahni exchange potential is also plotted in Fig. 3.

(v) In Sec. III and Appendix C, $\varepsilon_{x}(r)$ has been constructed in terms of (integrals on) $\rho(r)$ plus its derivatives and the kinetic-energy density $t(r)$. Reference may be made here to a recent condensed matter analogue, namely, the infinite barrier model of a metal surface (March [16]), where again $\varepsilon_{x}(r)$ is related to $t(r)$.

(vi) Scott's semiclassical calculation of the total exchange energy $\propto Z^{5 / 3}$ for heavy nonrelativistic neutral atoms is complemented by a study of the strong ionization limit $(N / Z) \ll 1$ in Appendix B. The result has the form $E_{x}^{\text {semiclassical }} \propto Z^{5 / 3}(N / Z)^{2 / 3}$, which becomes proportional to $Z$ for fixed (and still large) $N$. This then makes contact with

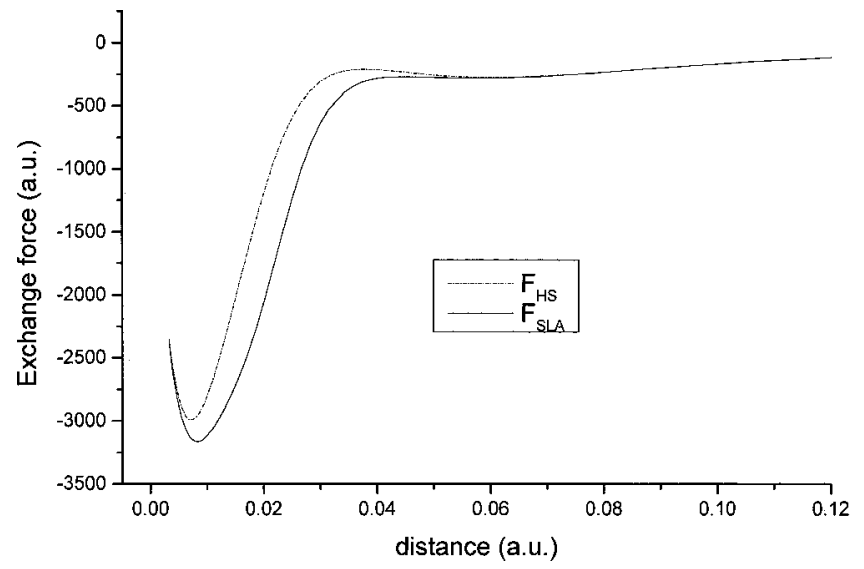

FIG. 4. (Radial) exchange forces $E_{\mathrm{HS}}(r)=-\partial V_{\mathrm{HS}}(r) / \partial r$ and $E_{\mathrm{Sla}}(r)=-\partial V_{\mathrm{Sla}}(r) / \partial r$ corresponding to the Harbola-Sahni and Slater exchange potentials, respectively. 
item (ii) above in the present section.

As to future directions, it would be of interest to extend the above to homonuclear diatomic molecules with a fixed number of electrons. However, unlike the bare Coulomb one-center problem treated here, the idempotent bare Coulomb density matrix for the two-center problem can no longer be obtained in closed analytic form (except near the united-atom limit for the present ten-electron one-center problem). Nevertheless, a study of the exchange energy $E_{x}(Z, N, R)$ for fixed (and small) $N$ is called for, at a number of values of the internuclear distance $R$.

\section{ACKNOWLEDGMENTS}

One of us (I.A.H.) wishes to acknowledge support from the Flemish Science Foundation (FWO) under Grant No. G0347.97. We also thank the University of Antwerp (RUCA) for its support. P.S. thanks the FWO for financial support. This work is also supported by the Concerted Action Program of the University of Antwerp.

\section{APPENDIX A: ANALYTIC FORMS OF ELECTRONIC, KINETIC-ENERGY AND EXCHANGE ENERGY DENSITIES FOR TEN-ELECTRON NONRELATIVISTIC ATOMIC IONS IN THE LIMIT OF LARGE $Z$}

From the study of March and Santamaria [9], who worked however with singly occupied states throughout, we find the electron density for the ten-electron atomic ions in the large- $Z$ limit as the diagonal element of the $1 \mathrm{DM} \gamma\left(r, r^{\prime}\right)$ given by Eq. (1.7) with

$$
F(r)=-(1 / 32 \pi)\left(Z / a_{0}\right)^{5} \exp \left(-Z r / a_{0}\right)
$$

and

$$
\begin{aligned}
\rho(r)= & (2 / \pi)\left(Z / a_{0}\right)^{3} \exp \left(-2 Z r / a_{0}\right) \\
& +(1 / 4 \pi)\left(Z / a_{0}\right)^{3} \exp \left(-Z r / a_{0}\right)\left[1-Z r / a_{0}\right. \\
& \left.+1 / 2\left(Z r / a_{0}\right)^{2}\right] .
\end{aligned}
$$

The corresponding kinetic-energy density $t(r)$ is then readily obtained from Eq. (1.2) as

$$
\begin{aligned}
t(r)= & \left(\hbar^{2} / 2 \pi m\right)\left(Z / a_{0}\right)^{5}\left\{2 \exp \left(-2 Z r / a_{0}\right)\right. \\
& \left.+(1 / 16) \exp \left(-Z r / a_{0}\right)\left[7-3 Z r / a_{0}+(1 / 2)\left(Z r / a_{0}\right)^{2}\right]\right\} .
\end{aligned}
$$

It is of interest to note that differentiating (A3), one can verify the equivalence with the result of Amovilli and March [22] that

$$
t^{\prime}(r)=(1 / 8) \rho^{\prime \prime \prime}-\left(3 / 4 r^{2}\right) \rho^{\prime}-\left(3 Z / 2 r^{2}\right) \rho .
$$

The basically new result of the present investigation is the exchange energy density $\varepsilon_{x}(r)$ calculated from Eq. (1.9) using the bare Coulomb density matrix (1.7), with the result in Eq. (2.2).
To conclude this appendix, we note that the total exchange energy $E_{x}$ and the force $-\nabla V_{x}(\mathbf{r})$ associated with the exchange potential $V_{x}(\mathbf{r})$ are connected by

$$
E_{x}=-\int \rho(\mathbf{r}) \mathbf{r} \cdot \nabla V_{x}(\mathbf{r}) d^{3} r
$$

a result that goes back to Levy and Perdew [23]. Equation (A5) is to be contrasted with the result for the exact $E_{x}$ in terms of the Slater potential, namely,

$$
E_{x}=\int \varepsilon_{x}(\mathbf{r}) d^{3} r=(1 / 2) \int \rho(\mathbf{r}) V_{\mathrm{Sla}}(\mathbf{r}) d^{3} r
$$

We note also that there is ambiguity in defining an exchange energy density. We have taken the definition in Eq. (1.9), which leads to $\varepsilon_{x}(r)$ in Eqs. (2.1) and (2.2). But one could adopt the (negative of) the integrand in Eq. (A5),

$$
4 \pi r^{2} \varepsilon_{x}^{\text {virial }}(r)=-\rho(r) r \frac{\partial V_{x}}{\partial r} 4 \pi r^{2}
$$

The area under this curve, namely, the total exchange energy $E_{x}$, is, of course, the same as that for $\left(4 \pi r^{2}\right) \varepsilon_{x}(r)$, with $\varepsilon_{x}(r)$ defined directly from the Dirac expression for $E_{x}[3]$.

\section{APPENDIX B: SEMICLASSICAL EVALUATION OF EXCHANGE ENERGY $E_{x}$ FOR HIGHLY CHARGED POSITIVE ATOMIC IONS IN THE LIMIT WHEN THE NUMBER OF ELECTRONS $N$ AND THE NUCLEAR CHARGE $Z$ ARE BOTH LARGE, BUT $N / Z \ll 1$}

The objective of this appendix is to evaluate the semiclassical approximation $E_{x}^{\text {semiclassical }} \equiv E_{x}^{\text {scl }}$ to the Dirac exchange energy [3]. This will be written explicitly as

$$
E_{x}^{\mathrm{scl}}=-c_{x} \int\left\{\rho_{\mathrm{TF}}(r)\right\}^{4 / 3} d^{3} r, \quad c_{x}=(3 / 4)(3 / \pi)^{1 / 3} e^{2}
$$

The Thomas-Fermi semiclassical density $\rho_{\mathrm{TF}}(r)$ for a bare Coulomb potential is immediately written down from phasespace considerations as

$$
\rho_{\mathrm{TF}}(r)=\left(8 \pi / 3 h^{3}\right)(2 m)^{3 / 2}\left(\mu+Z e^{2} / r\right)^{3 / 2},
$$

where the chemical potential $\mu$ is found from

$$
\int \rho_{\mathrm{TF}}(r) d^{3} r=N
$$

to be

$$
\mu=-18^{-1 / 3}\left(Z^{2} / N^{2 / 3}\right)\left(e^{2} / a_{0}\right)
$$

with $a_{0}=\hbar^{2} / m e^{2}$. Evidently Eq. (B2) is to be used from $r$ $=0$ out to the semiclassical radius $r_{0}$ given by

$$
\mu=-Z e^{2} / r_{0}
$$

and then one finds from Eqs. (B1), (B2), and (B5) the result 


$$
\begin{aligned}
E_{x}^{\mathrm{scl}} & =-c_{x}\left(8 \pi / 3 h^{3}\right)(2 m)^{2} \int_{0}^{r_{0}}\left(Z e^{2}\right)^{2}\left[1 / r-1 / r_{0}\right]^{2} 4 \pi r^{2} d r \\
& =-c_{x}\left(8 \pi / 3 h^{3}\right)(2 m)^{2}\left(Z e^{2}\right)^{2}\left(4 \pi r_{0} / 3\right) .
\end{aligned}
$$

Substitution for $c_{x}$ from Eq. (B1) and for $r_{0}$ from Eqs. (B4) and (B5) then yields the desired result

$$
E_{x}^{\mathrm{scl}}=-\left(4 / 3 \pi^{2}\right)\left(18 N^{2}\right)^{1 / 3} Z e^{2} / a_{0} .
$$

As evidenced in the title of this appendix, Eq. (B7) is physically valid when both $Z$ and $N$ are large, but $N / Z \ll 1$.

In the text, we have noted the "exact" evaluation of the LDA exchange in Eq. (B1) with the correct electron density for ten electrons in a bare Coulomb field. As in Eq. (B7), $E_{x}^{\mathrm{LDA}} \propto Z$, but, of course, $N=10$. Then Eq. (B7) is being used outside its proper range of validity. Thus, substituting $N$ $=10$ in Eq. (B7) gives $-1.6433 Z\left(e^{2} / a_{0}\right)$, to be compared with $E_{x}^{\mathrm{LDA}}=-1.6084 Z\left(e^{2} / a_{0}\right)$.

It is worth noting also that Eq. (B7) complements the exchange energy calculated by Scott [10] for neutral atoms; he found $-0.221 Z^{5 / 3}\left(e^{2} / a_{0}\right)$ with the self-consistent Thomas-Fermi neutral atom density; Eq. (B7) would give $\approx-0.354 Z^{5 / 3}\left(e^{2} / a_{0}\right)$ due to neglect of screening, which is permissible only for $(N / Z) \ll 1$, with $N$ and $Z$ large.

\section{APPENDIX C: EXCHANGE ENERGY IN TERMS OF ELECTRON AND KINETIC-ENERGY DENSITIES}

The total exchange energy $E_{x}=E_{x}^{(1)}+E_{x}^{(2)}+E_{x}^{(3)}$, where the simplest term $E_{x}^{(1)}$ is given explicitly by

$$
\begin{aligned}
E_{x}^{(1)}= & -\left(2 \pi^{2} e^{2}\right) \int_{0}^{\infty} d r r \int_{0}^{\infty} d r^{\prime} r^{\prime} \\
& \times\left[\left(r+r^{\prime}\right)-\left|r-r^{\prime}\right|\right] \rho^{2}\left(\left(r+r^{\prime}\right) / 2\right) .
\end{aligned}
$$

The change of variable defined by

$$
s=\left(r+r^{\prime}\right) / 2
$$

then allows $E_{x}^{(1)}$ to be rewritten as

$$
\begin{aligned}
E_{x}^{(1)}= & -4 \pi^{2} e^{2} \int_{0}^{\infty} d r r\left[\int_{r / 2}^{r} 2 d s(2 s-r)^{2} \rho^{2}(s)\right. \\
& \left.+\int_{r}^{\infty} 2 d s r(2 s-r) \rho^{2}(s)\right] .
\end{aligned}
$$

Introducing the Heaviside function $H(a, b)$ defined by

$$
\begin{aligned}
H(a, b) & =1, & & b>a \\
& =0, & & 0<b<a
\end{aligned}
$$

one can interchange the order of integration in Eq. (C3) to obtain

$$
\begin{aligned}
E_{x}^{(1)}= & -8 \pi^{2} e^{2}\left\{\int_{0}^{\infty} d s \rho^{2}(s) \int_{0}^{\infty} d r r(2 s-r)^{2} H(r / 2, s)\right. \\
& \times[1-H(r, s)]+\int_{s=0}^{\infty} d s \rho^{2}(s) \int_{r=0}^{\infty} d r r^{2} \\
& \times(2 s-r) H(r, s)\} .
\end{aligned}
$$

The $r$ integration is readily performed to find

$$
E_{x}^{(1)}=-\left(20 \pi^{2} e^{2} / 3\right) \int_{0}^{\infty} d s s^{4} \rho^{2}(s)
$$

By an analogous procedure, $E_{x}^{(2)}$ and $E_{x}^{(3)}$ may be calculated, with the results

$$
E_{x}^{(2)}=-\left(224 \pi^{2} e^{2} / 9\right) \int_{0}^{\infty} d s \rho(s) F(s) s^{6}
$$

and

$$
E_{x}^{(3)}=-\left(32 \pi^{2} e^{2}\right) \int_{0}^{\infty} d s F^{2}(s) s^{8} .
$$

Inserting the forms (A2) and (A1) for $\rho$ and $F$, respectively, into the definite integrals in Eqs. (C6) $-(\mathrm{C} 8)$ yields the results

$$
\begin{gathered}
E_{x}^{(1)}=-(18355 / 3888) Z e^{2} / a_{0}, \\
E_{x}^{(2)}=(760585 / 139968) Z e^{2} / a_{0},
\end{gathered}
$$

and

$$
E_{x}^{(3)}=-(315 / 128) Z e^{2} / a_{0},
$$

with the final result for the total exchange energy $E_{x}$ given in Eq. (2.3).
[1] R. G. Parr and W. Yang, Density Functional Theory of Atoms and Molecules (Oxford University Press, Oxford, 1989).

[2] A. Holas and N. H. March, Phys. Rev. A 51, 2040 (1995).

[3] P. A. M. Dirac, Proc. Cambridge Philos. Soc. 26, 376 (1930).

[4] N. H. March and R. J. White, J. Phys. B 5, 466 (1972).

[5] E. A. Hylleraas, Z. Phys. 65, 209 (1930).

[6] See, for example, D. Layzer, Ann. Phys. (N.Y.) 8, 271 (1959); or the later review of M. Cohen, Adv. At. Mol. Phys. 25, 195
(1988).

[7] See, e.g., the review by N. H. March, Adv. Phys. 6, 1 (1957); N. H. March, Self-Consistent Fields in Atoms (Pergamon, Oxford, 1975).

[8] Y. Tal and M. Levy, Phys. Rev. A 23, 408 (1981).

[9] N. H. March and R. Santamaria, Phys. Rev. A 38, 5002 (1988).

[10] J. M. C. Scott, Philos. Mag. 43, 859 (1951). 
[11] N. H. March and R. G. Parr, Proc. Natl. Acad. Sci. USA 77, 6285 (1980).

[12] N. H. March and P. Wind, Mol. Phys. 77, 791 (1992).

[13] S. Kais, S. M. Sung, and D. R. Herschbach, Int. J. Quantum Chem. 49, 657 (1994).

[14] M. K. Harbola and V. Sahni, Phys. Rev. Lett. 62, 489 (1989).

[15] M. Levy and N. H. March, Phys. Rev. A 55, 1885 (1997).

[16] N. H. March, Phys. Rev. B 60, 5011 (2000).

[17] N. H. March and R. Santamaria, Int. J. Quantum Chem. 39,
585 (1991).

[18] H. Lee, C. Lee, and R. G. Parr, Phys. Rev. A 44, 768 (1991).

[19] G. K-L. Chan and N. C. Handy, Phys. Rev. A 59, 2670 (1999).

[20] N. H. March, Phys. Lett. A 114, 301 (1986); see also A. Nagy and N. H. March, Chem. Phys. Lett. 181, 279 (1991).

[21] L. Kleinman, Phys. Rev. B 49, 14197 (1994).

[22] C. Amovilli and N. H. March, Phys. Chem. Liq. 38, 505 (2000).

[23] M. Levy and J. Perdew, Phys. Rev. A 32, 2010 (1985). 